\title{
Diagnostic efficacy of risk of malignancy index in adnexal mass: a prospective study
}

\author{
Anusha Kamath, Satyam Satyarth, Pariseema Dave \\ Corresponding author: Dr Anusha Kamath, Assistant Professor, Department of Obstetrics and \\ Gynaecology, AIIMS, Nagpur, India; Email - dranushakamath@gmail.com
}

Distributed under Attribution-Non Commercial - Share Alike 4.0 International (CC BY-NC-SA 4.0)

\begin{abstract}
Objectives: The purpose of this study was to determine if the risk of malignancy index (RMI 2) can distinguish between benign and malignant adnexal masses in the women with adnexal masses. Methods: This is a prospective observational study conducted over a period of two years from 2012 to 2014. A total of 100 cases of adnexal mass were selected using purposive sampling technique. Information regarding age, parity, menstrual status, history of previous surgery and symptoms were noted. RMI 2 was calculated for each patient. Statistical analysis was done using SPSS software version 21. Results: Majority (60\%) of the women were in the perimenopausal or menopausal age group (40-60 years). Majority of the patients ie $62 \%$ had levels of CA- 125 above100U/1, 16\% had levels between 35 $100 \mathrm{U} / 1$ and $22 \%$ had levels $<35 \mathrm{U} / 1$. RMI was greater than 200 in $72 \%$ patients indicating malignancy and was less than 200 in $28 \%$ of the patients suggesting benign disease. The sensitivity of the RMI for diagnosing malignant lesions was $84 \%$ while the specificity was $67 \%$. The PPV was $89 \%$ and the NPV was $57 \%$. Conclusion: RMI appears to be a valuable, reliable and applicable method in the primary evaluation of patients with pelvic masses and a usable method in referral of relevant patients for centralised surgical treatment.
\end{abstract}

Keywords: RMI, ovarian cancer, referral.

Ovarian cancer is the fourth most common malignancy in females and the second most common gynaecological malignancy after cervical cancer ${ }^{1}$. Epithelial ovarian cancer has the highest fatality to case ratio of all the gynaecological malignancies because more than two-thirds of patients have advanced disease at diagnosis ${ }^{2}$. It presents a major surgical challenge, requires intensive and often complex therapies, and is extremely demanding of the patient's psychological and physical energy.

Ovarian cancer most commonly presents as adnexal mass. Adnexal abnormalities may be discovered as a result of screening, be an incidental finding, or be recognized as a result of investigations performed specifically for a suspected pelvic mass ${ }^{3}$. The only definitive way of determining whether a mass is benign or malignant is removal at surgery. However, the majority of women with adnexal masses will not have malignant disease and many do not require surgery. Those women who are likely to have ovarian cancer should be referred to specialized oncology centres with ability to perform optimal surgical staging and cytoreduction ${ }^{4,5}$.

The discrimination between benign and malignant adnexal masses is central to decisions regarding clinical management and surgical planning in such patients. Patients with malignant tumors should be referred to a gynecological oncologist, as the quality of cytoreductive surgery and

Received: $24^{\text {th }}$ March 2020. Accepted: $3^{\text {rd }}$ May 2020.

Kamath A, Satyarth S, Dave P. Diagnostic efficacy of risk of malignancy index in adnexal mass: a prospective study. The New Indian Journal of OBGYN. 2020; 7(1): 4-9. 
The New Indian Journal of OBGYN. 2020 (July-December);7(1)

surgical staging/lymph node dissection are important prognostic factors in ovarian cancer ${ }^{4,5}$. The extent of cytoreductive surgery is associated with the specific skills and experience of well-trained gynaecologic oncologists hence, improving the prognosis and five year survival ${ }^{6}$. Furthermore, appropriate and timely referral to a gynecological oncologist has been proven to increase survival in patients with ovarian cancer ${ }^{7}$. Streamlining of referrals in oncology has always been a clinical challenge in attempting to create a satisfactory safety net.

Pelvic assessment, tumor markers, and radiological investigations have been proposed in this regard, but all of the parameters when considered separately, are inadequately sensitive or specific. Various methods of evaluating ovarian mass have also been proposed ${ }^{8}$. Risk of malignancy index (RMI) is a combined parameter, which is simple, practical and highly sensitive, and more specific. RMI is calculated with a simplified regression equation obtained from the product of menopausal status score (M), ultrasonography score (U), and absolute value of serum CA-125 ${ }^{9}$. Jacobs et al (1990) was first to devise RMI 1 which utilized the ultrasound findings, menopausal status and serum CA 125 levels to predict the risk of malignancy with greater sensitivity and specificity than any one factor alone. Later, it was adjusted by Tingulstad et al in 1996 as RMI 2 and again modified in 1998 as RMI 3. Yamamoto et al created their own model of a malignancy risk index. They added the parameter of the tumour size (S) to the RMI and have termed it the RMI $4^{10-12}$. Some of the potential advantages of RMI include rapid triage of patients through the referral system. Early identification of ovarian carcinomas and referral to a gyneco-oncologist can facilitate accurate staging of the disease and optimal cytoreductive treatment, enhancing patient survival ${ }^{13}$. The purpose of this study was to determine if the RMI (RMI 2) can distinguish between benign and malignant adnexal masses in the population of women referred to the Gujarat Cancer and Research Institute.

\section{Materials and methods}

This is a prospective observational study conducted over a period of two years from 2012 to 2014. A total of 100 cases of adnexal mass were selected using purposive sampling technique. All the patients were admitted to the Department of Gynaec-oncology at G.C.R.I., Ahmedabad. Approval was obtained from the ethics committee of the institution.

All women presenting with adnexal masses scheduled for surgery were recruited for the study. Women already diagnosed cases with ovarian malignancy receiving chemotherapy, masses arising from urinary tract and gastrointestinal tract and pregnancy with its complications like ectopic, molar and post abortive were excluded from the study. Apart from the demographic information, information regarding age, parity, menstrual status, history of previous surgery and symptoms were noted. All patients underwent routine physical examination.

Apart from the routine pre-operative investigations, menopausal status, abdominal ultrasound findings and preoperative serum CA 125 levels were recorded. Serum levels of CA-125 were measured by Electrochemiluminescence immunoassaay (ECLIA) method. An ultrasonographic evaluation of their pelvic mass for each of the following characteristics: multilocularity (more than bilocular), presence of solid areas, bilaterality, presence of ascites, and extra ovarian tumors or evidence of metastases. For each ultrasonographic characteristic a score of one was assigned and a total ultrasound score (U) was calculated. Postmenopausal status (M) was defined as more than one year of amenorrhea or an age of more than 50 years in women who have had a hysterectomy. The modified RMI (RMI 2) for each woman was calculated using the product of the ultrasound score (U), the menopausal score (M), and the absolute value of serum CA-125 inserted in the following formula:

$\mathrm{RMI}=\mathrm{U} \times \mathrm{M} \times$ Serum $\mathrm{CA}-125$

For analysis purposes, tumours of low malignant potential were classified as malignant because it was considered ideal for these tumours to be surgically managed by a gynaecologic oncologist and the final histopathology was regarded as the true definite outcome. Staging laparotomy was performed for all patients. The type of surgical procedure done were either unilateral salpingooophorectomy, unilateral salpingo-oophorectomy with biopsy of the contralateral ovary, total abdominal hysterectomy and unilateral salpingo-oophorectomy, total abdominal hysterectomy with bilateral salpingooophorectomy, with omentectomy, with bilateral pelvic lymph node dissection and debulking surgery. Surgical staging was carried out in suspected malignant ovarian tumors. The pelvic and para-aortic lymph nodes were evaluated and all enlarged lymph node were resected. Infracolic omentectomy was performed. The other operative findings recorded were gross appearance and cut surface, ascites, site of extra ovarian involvement and tumor size. 
The New Indian Journal of OBGYN. 2020 (July-December);7(1)

Specialist gynaecological oncology surgeons at GCRI performed all surgical procedures. Histopathological examination of all specimens were done in the department of pathology at GCRI by a specialist in gynaecological pathology. The tumours were classified according to the WHO classification. Malignant tumours were staged according to the FIGO staging system. Statistical analysis was done using SPSS software version 21. A univariate statistical analysis was performed for all sonographic parameters and patient age.

\section{Results}

During the two year period, 100 women who met the inclusion criteria were enrolled in the study. Majority $(60 \%)$ of the women were in the perimenopausal or menopausal age group ( $40-60$ years). Only $7 \%$ are under the age of 20 years and $13 \%$ above the age of 60 years (table 1). Twenty percent of the women were in the age group of 21-40 years.

Table 1: Age distribution

\begin{tabular}{lll}
\hline $\begin{array}{l}\text { Age in } \\
\text { years }\end{array}$ & $\begin{array}{l}\text { Number of patient } \\
(\mathbf{N}=\mathbf{1 0 0})\end{array}$ \\
\hline & Benign & Malignant \\
\cline { 2 - 3 }$<20$ & 04 & 03 \\
$20-40$ & 14 & 06 \\
$40-60$ & 17 & 43 \\
$>60$ & 05 & 08
\end{tabular}

The median age was 48 years. A large proportion (62\%) of the women were menopausal, $36 \%$ were menstruating and only 2 were premenarchal. Parity is an important etiological factor and greater parity affords greater protection against ovarian cancer. In our study, $1 \%$ were nulliparous, $75 \%$ were multiparous and $9 \%$ were unmarried. In nulliparous patients and patients with one child, future child bearing was given due consideration and fertility preserving surgery was carried out wherever possible.

The most common complaint was abdominal pain (63\%) followed by abdominal distension (40\%), abdominal mass $(38 \%)$ and nonspecific complaints of vomiting and anorexia (25\%). Majority of the patients ie $62 \%$ had levels of CA- 125 above $100 \mathrm{U} / 1,16 \%$ had levels between $35-100 \mathrm{U} / 1$ and $22 \%$ had levels $<35 \mathrm{U} / 1$. Upon analysing, out of 24 benign cases 18 had serum CA-125 values well within the normal limits whereas 6 had levels more than 35. Among the 76 malignant cases, only five had levels below 35 that they were false negative whereas majority of the cases (71) had elevated levels. RMI was greater than 200 in $72 \%$ patients indicating malignancy and was less than 200 in $28 \%$ of the patients suggesting benign disease. Majority (65\%) had ultrasound scoring of more than 2 suggesting that the mass is malignant

Table 2: Histopathological co-relation of malignant adnexal mass with RMI

\begin{tabular}{llll}
\hline HPE type & & RMI<200 & RMI $\mathbf{2 0 0}$ \\
\hline Serous & Borderline & - & 1 \\
& Malignant & 5 & 46 \\
Mucinous & Borderline & 2 & 2 \\
& Malignant & 1 & 5 \\
Germ cell tumour & & 1 & 1 \\
Sertoli cell tumour & - & 1 \\
Transitional cell carcinoma & - & 1 \\
Granulosa cell tumour & 1 & 3 \\
Adenofibrocarcinoma & & - & 1 \\
Seromucinous tumour & Sigmoid & - & 2 \\
Metastatic carcinoma & 1 & 2 \\
Total & Breast & - & 1 \\
\hline
\end{tabular}

in nature. The histopathological co-relation of malignant masses with RMI is shown table 2. It shows that majority of the cases were serous adenocarcinoma (81\%), 1 borderline and 51 malignant. Ten were of mucinous variety out of which 4 were borderline and 6 were malignant. Four were metastatic from other sites, 3 being from sigmoid and 1 from

Table 3: False positive cases in benign cases

\begin{tabular}{|c|c|}
\hline Histopathological diagnosis & Number \\
\hline Mucinous tumour & 2 \\
\hline Teratoma & 2 \\
\hline Benign cyst & 2 \\
\hline Infective Tuberculosis & 1 \\
\hline Salpingo-oophoritis & 1 \\
\hline
\end{tabular}

breast. Among benign cases, majority were teratoma (29\%) of which 5 had RMI $<200$ and 2 had RMI more than 200.5 were of mucinous variety out of which 3 had RMI $<200$. Five were hemorrhagic cysts and 4 were infective in nature.

Table 4: False negative cases in malignant cases

\begin{tabular}{ll}
\hline Histopathological diagnosis & Number \\
\hline Borderline mucinous tumour & 2 \\
Malignant mucinous tumour & 1 \\
Germ cell tumour & 1 \\
Metastatic tumour from sigmoid & 1 \\
Granulosa cell tumour & 1 \\
\hline
\end{tabular}
Among the infective cases, 3 were of tuberculosis and 1 was of salpingo-oophoritis. Out of eight false positive cases, 2 were mucinous tumour, 2 were teratoma, 2 were benign cyst and 2 were of infective type (table 3). Solid parts found in dermoid cysts and multilocular cystic lesions found in mucinous cystadenomas may attribute to the false positive cases. Out of the six false negative cases shown in table 4, 2 were of borderline mucinous variety, 1 was malignant 
mucinous, 1 was germ cell tumour and 1 each were metastatic tumour from sigmoid and granulosa cell tumour.

Table 5: RMI in adnexal masses

\begin{tabular}{lll}
\hline RMI & Benign & Malignant \\
\hline$<200$ & $16(57 \%)$ & $12(43 \%)$ \\
$>200$ & $8(11 \%)$ & $64(89 \%)$ \\
\hline
\end{tabular}

There may be limitation of RMI in detecting borderline cases. In patients with RMI $>200,89 \%$ were malignant and $11 \%$ were benign whereas in those with RMI $<200,43 \%$ were malignant and $57 \%$ were benign (table 5). The

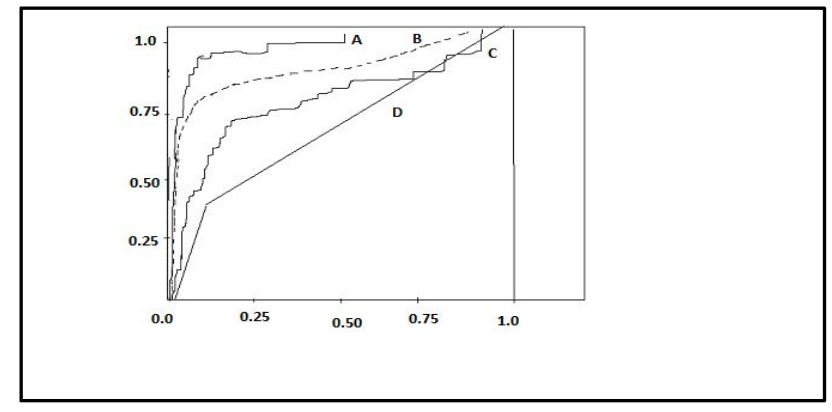
A- Risk of Malignancy Index
B- Ultrasound Score
C- CA- 125
D- Menopausal status

Figure 1: Receiver operating curves (ROC) showing the diagnostic performances of $\mathrm{CA}-125$, ultrasonographic scores and the risk of malignancy index.

sensitivity of the RMI for diagnosing malignant lesions was $84 \%$ while the specificity was $67 \%$. The PPV was $89 \%$ and the NPV was $57 \%$. The figure-1 shows receiver operating curves showing the diagnostic performances of CA-125, ultrasonographic scores and the risk of malignancy index of the patients. The ROC curves were constructed to determine the appropriate cut-off value for discriminating benign from borderline and malignant tumors.

\section{Discussion}

Currently, it appears that the best way to detect early ovarian cancer for both the patient and her clinician to have a high index of suspicion of the diagnosis in the symptomatic woman ${ }^{5}$. The discrimination between benign and malignant adnexal masses is central to decisions regarding clinical management and surgical planning in such patients. Several studies have shown that ovarian cancer patients who underwent surgery by a gynecologist had better survival compared with patients who underwent surgery by general surgeons ${ }^{14,15}$. Subsequently, it was suggested that surgery by a gynecologic oncologist would improve survival further ${ }^{16-18}$. A standardized method for preoperative identification of probable malignant masses would allow optimization of first-line treatment for women with ovarian cancer ${ }^{19}$. Unfortunately, no single method accurately predicts ovarian malignancy at present. A scoring system that would help to predict malignancy would aid in proper counseling and timely referral to a specialized centre.

In this study, we aim to determine if the RMI (RMI 2) can distinguish between benign and malignant adnexal masses. In our study, 100 cases of adnexal masses were analysed and risk of malignancy was calculated and various parameters were studied. All the patients underwent treatment as per standards of care given by $\mathrm{NCCN}$ and FIGO. We found that the mean age of the patients was 48 years which is higher than that of the study by Tahereh et al. Ovarian cancer was most common in the age group of 41-60 years ${ }^{20}$. The US score appeared to be the most useful in discriminating benign and malignant ovarian tumours ${ }^{21,22}$. A score of more than 7 yielded the maximal area under the curve and would be the best indicator of malignant nature of the ovarian mass with the highest sensitivity and specificity. Several retrospective and prospective studies have reported RMI to be the best available tool for triage and referral of ovarian malignancies 23,24 .

In our study, out of the 76 patients, only 12 had RMI less than 200 with a positive predictive value of nearly $90 \% .62 \%$ of the patients in our study were menopausal which is similar to incidence rates reported in other studies ${ }^{25-27}$. Among the 76 malignant cases, only five had levels below 35 that is they were false negative whereas majority of the cases (71) had elevated levels. This corroborates with the findings of the study published in J Med Assoc Thai Vol. 88 Suppl 22005 in which the level was $>35 \mathrm{U} / \mathrm{ml}$ in $23 \%$ and $89 \%$ of benign and malignant ovarian tumours respectively ${ }^{28}$. Most studies reported an increased diagnostic accuracy and performance with an RMI cut-off of $200^{9-12,24,26}$. A recent study reported a sensitivity of $89.5 \%$, specificity of $96.2 \%$, positive predictive value of $77.3 \%$, and negative predictive value of 98.4\% when a higher RMI cut-off of 238 was used for the screening ${ }^{20}$. Yamamoto et al. (2009) reported a sensitivity and specificity of $75 \%$ and $91 \%$, respectively, using a cut-off of $450{ }^{29}$. There also seems to be limitation of RMI in detecting borderline cases but more studies are required to establish the relation ${ }^{30}$. The sensitivity of RMI as calculated 
The New Indian Journal of OBGYN. 2020 (July-December);7(1)

by this study is $84 \%$, specificity being $67 \%$ and the positive and negative predictive value $89 \%$ and $57 \%$ respectively.

As opposed to this, the study by Jacobs et al gave a sensitivity of $85.4 \%$ with a specificity of $96.9 \%{ }^{11}$ whereas Tahereh et al $89.5 \%$ and $94.7 \%{ }^{20}$ respectively. Previous studies (Tingulstad's et al) showed a reduced sensitivity of RMI for lesions of borderline malignancy ${ }^{12}$. Comparable results are seen in our study. However, in all studies, the number of borderline tumors has been small.

\section{Conclusion}

In conclusion, the present study has demonstrated RMI to be a valuable, reliable and applicable method in the primary evaluation of patients with pelvic masses and a usable method in referral of relevant patients for centralised surgical treatment. A very strong argument for the use of RMI in the primary evaluation of patients with pelvic masses is the simplicity of the method and its easy applicability. This study confirms its accuracy in detecting malignancy but also highlights its limitations in excluding benign diseases.

\section{Conflict of interest: None. Disclaimer: Nil.}

\section{References}

1. Consolidated Report of Population Based Cancer Registries 2001-2004. National Cancer Registry Program. Bangalore: Indian Council of Medical Research; 2006.

2. Rossing MA, Wicklund KG, Cushing-Haugen KL, Weiss NS. Predictive value of symptoms for early detection of ovarian cancer. J Natle Cancer Inst. 2010; 102(4):222-9.

3. Burbos N, Duncan TJ. Management of a pelvic mass. Obstetrics, Gynaecology \& Reproductive Medicine. 2010; 20(11): 335-40.

4. Giede KC, Kieser K, Dodge J, Rosen B. Who should operate on patients with ovarian cancer? An evidencebased review. Gynecol Oncol. 2005; 99(2): 447-61.

5. ACOG Committee Opinion number 280. The role of the generalist obstetrician gynecologist in the early detection of ovarian cancer. Gynecol Oncol. 2002; 2002(100): 1413-6.

6. Eisenkop SM, Spirtos NM, Montag TW, Nalick RH, Wang HJ. The impact of subspecialty training on the management of advanced ovarian cancer. Gynecol Oncol. 1992; 47: 203-9.

7. Kirwan JM, Tinchello DG, Herodd JJ, Frost O, Kingston RE. Effect of delays in primary care referral on survival of women with epithelial ovarian cancer. BMJ. 2002; 324(7330): 148-51.

8. Twickler DM, Forte TB, Santos-Ramos R, McIntire D, Harris P, Scott D. The Ovarian Tumor Index predicts risk for malignancy. Cancer. 1999; 86: 2280-90

9. Akturk E, Karaka RE, Alanbay I, Dede M, Karaşahin E, Yenen $\mathrm{MC}$, et al. Comparison of four malignancy risk indices in the detection of malignant ovarian masses. $\mathrm{J}$ Gynecol Oncol. 2011; 22(3):177-82.

10. Morgante G, Marca AI, Ditto A, De Leo V. Comparison of two malignancy risk indices based on serum CA-125, ultrasound score and menopausal status in the diagnosis of ovarian masses. Br J Obstet Gynecol. 1999; 106(6): $524-7$

11. Jacobs I, Oram D, Fairbanks J, Turner J, Frost C, Grudzinskas JG. A risk of malignancy index incorporating CA-125, ultrasound and menopausal status for the accurate pre-operative diagnosis of ovarian cancer. Br J Obstet Gynecol. 1990; 97(10): 922-7.

12. Tingulstad S, Hagen B, Skjeldestad FE, Halvorsen T, Nustad K, Onsrud M. The Risk-of- Malignancy Index to evaluate potential ovarian cancers in local hospitals. Obstet Gynecol. 1999; 93(3): 448-52.

13. Bristow RE, Tomacruz RS, Armstrong DK, Trimble EL, Montz FJ. Survival effect of maximal cytoreductive surgery for advanced ovarian carcinoma during the platinum era: ametaanalysis. J Clin Oncol. 2002; 20(5): 1248-59.

14. Bailey J, Tailor A, Naik R, Lopes A, Godfrey K, Hatem $\mathrm{HM}$, et al. Risk of Malignancy Index for Referral of Ovarian Cancer Cases to a Tertiary Center: Does It Identify the Correct Cases?. Int J of Gynecol cancer. 2006; 16 (Suppl 1): 30-4

15. Anderson ES, Knudsen A, Rix P, Johansen B. Risk of Malignancy Index in the Preoperative Evaluation of Patients With Adnexal Masses. Gynecol Oncol. 2003; 90 (1): 109-12

16. Kos HE, Willemse PH, Aalders JG, de Vries EG, Schaapveld M, Otter R, et al. Surgery by consultant gynecologic oncologists improves survival in patients with ovarian carcinoma. Cancer 2006 Feb; 106(3): 58998.

17. Grabowski JP, Harter P, Buhrmann C, Lorenz D, Hils R, Kommoss S, et al. Re-operation outcome inpatients referred to a gynecologic oncology center with presumedovarian cancer FIGO I-IIIA after sub-standard initial surgery. Surg Oncol. 2012 Mar; 21(1): 31-5. 
18. Kamath A, Dave P, Mankad M. Evaluation of Incompletely Staged Ovarian Malignancy: A Study in Western India. Journal of South Asian Federation of Menopause Societies. 2017; 5(2):133-7.

19. Rujuta J, Maitra N. Risk of malignancy index (RMI) in evaluation of adnexal mass. The Journal of Obstetrics and Gynecology of India. 2015; 65(2): 117-21

20. Ashrafgangooei T, Rezaeezadeh M. Risk of Malignancy Index in Preoperative Evaluation of Pelvic Masses. Asian Pacific Journal of Cancer Prevention. 2011; 12(7): 1727-30.

21. Rein BJD, Gupta S, Dada R, Safi J, Michener C, Agarwal A. Potential Markers for Detection and Monitoring of Ovarian Cancer. Journal of Oncology. 2011; 2011: Article ID 475983

22. Ferrazzi E, Zanetta G, Dordoni D, Berlanda N, Mezzopane R, Lissoni AA. Transvaginal ultrasonographic characterization of ovarian masses: comparison of five scoring systems in a multicenter study. Ultrasound Obstet Gynecol. 1997; 10: 192-7.

23. Davis AP, Jacobs 1, Wools R, Fish A, Oram D. The adnexal mass: benign or malignant? Evaluation of a risk of malignancy index. $\mathrm{Br} \mathrm{J}$ Obstet Gynaecol. 1993; 100(10): 927-31.

24. Geomini P, Kruitwagen R, Bremer GL, Cnossen J, Mol BWJ. The accuracy of risk scores in predicting ovarian malignancy: a systematic review. Obstet Gynecol. 2009; 113(2): 384-94.

25. Akdeniz N, Kuyumcuoğlu U, Kale A, Erdemoğlu M, Caca F. Risk of malignancy index for adnexal masses. Eur J Gynaecol Oncol. 2009; 30(2): 178-80.
26. Bouzari Z, Yazdani S, Kelagar ZS, Abbaszadeh N. Risk of malignancy index as an evaluation of preoperative pelvic mass. Caspian J Intern Med. 2011; 2(4): 331-5.

27. Dora SK, Dandapat AB, Pande B, Hota JP. A prospective study to evaluate the risk malignancy index and its diagnostic implication in patients with suspected ovarian mass. J Ovarian Res. 2017; 10(1): 55.

28. Leelahakorn S, Tangjitgamol S, Manusirivithaya S, Thongsuksai P. Comparison of ultrasound score, CA125, menopausal status, and risk of malignancy index in differentiating between benign and borderline or malignant ovarian tumors. J Med Assoc Thai. 2005; 88: 22-30.

29. Yamamoto Y, Yamada R, Oguri H, Maeda N, Fukaya T. Comparison of four malignancy risk indices in the preoperative evaluation of patients with pelvic masses. Eur J Obstet Gynecol Reprod Biol. 2009; 144(2): 163-7.

30. Meray O, Türkçüoğlu I, Meydanlı MM, Kafkaslı A. Risk of malignancy index is not sensitive in detecting non-epithelial ovarian cancer and borderline ovarian tumor. J Turk Ger Gynecol Assoc. 2010; 11(1): 22-6.

Anusha Kamath $^{1}$, Satyam Satyarth ${ }^{2}$, Pariseema Dave ${ }^{3}$

${ }^{1}$ Assistant Professor, Department of Obstetrics and Gynaecology, AIIMS, Nagpur, India; ${ }^{2}$ Consultant

Medical Oncologist, National Cancer Institute, Nagpur, India; ${ }^{3}$ Professor, Department of Gynaecologic Oncology, GCRI, Ahmedabad, India. 